\title{
Erratum to: Nucleofection of whole murine retinas
}

\author{
Iria Maria Gomez-Touriño • Ana Senra • \\ Francisco Gonzalez
}

Published online: 22 January 2013

(C) Springer Science+Business Media Dordrecht 2013

\section{Erratum to: Cytotechnology DOI 10.1007/s10616-012-9509-3}

Unfortunately, one of the co-authors' (Francisco Gonzalez) name has been incorrectly published in the original publication of the article.
The correct name should read as Francisco Gonzalez.

The online version of the original article can be found under doi:10.1007/s10616-012-9509-3.

I. M. Gomez-Touriño · A. Senra · F. Gonzalez

CIMUS (Department of Physiology), School of Medicine, University of Santiago de Compostela, Avd. Barcelona,

15782 Santiago de Compostela, Spain

Present Address:

I. M. Gomez-Touriño ( $\square)$

Department of Biochemistry and Molecular Biology,

School of Pharmacy, University of Santiago de

Compostela, Campus Vida s/n. C.P., 15782 Santiago de

Compostela, Spain

e-mail: iria.gomez.tourino@gmail.com

F. Gonzalez

Service of Ophthalmology, Complejo Hospitalario

Universitario de Santiago de Compostela, c/Dr. Baltar s/n, 15706 Santiago de Compostela, Spain 